\title{
Expression analysis of human intersectin 2 gene (ITSN2) minor splice variants showing differential expression in normal human brain
}

\author{
MARKUS SEIFERT $^{1,2}$, CHRISTINA AMPOFO $^{1}$, YASMIN MEHRAEIN ${ }^{1}$, \\ JÖRG REICHRATH ${ }^{2}$ and CORNELIUS WELTER ${ }^{1}$ \\ ${ }^{1}$ Department of Human Genetics, Saarland University, University Hospital; \\ ${ }^{2}$ Department of Dermatology, The Saarland University Hospital, Homburg/Saar, Germany
}

Received July 10, 2006; Accepted October 2, 2006

\begin{abstract}
Human intersectins 1 and 2 (ITSN1 and ITSN2) are conserved proteins involved in clathrin-mediated endocytosis. In both, two major splice variants, the so-called long and short isoforms have been identified. Whereas most analyses so far focussed on ITSN1, little is known about ITSN2. Data from expression analyses for the intersectin genes mainly refer to the major isoforms. Only recently have a few minor splice variants of ITSN2 been described, though no detailed analyses of their expression in different tissues have been performed. Using RT-PCR-studies we analyzed ITSN2 minor splice variants and their expression in an adult tissue panel. Thereby we demonstrated at least one new minor variant lacking exon 7. Differential expression was demonstrated for a previously described minor splice variant including exon 16 (ITSN2C) with a relative increase in adult human brain tissue. Additional comparative expression analyses in oligodendrogliomas furthermore revealed differential expression with lack of this specific minor splice variant in the brain tumor tissue. These results indicate that ITSN2C may be specifically expressed in neurons hinting to a physiologic role in neuronal cell function.
\end{abstract}

\section{Introduction}

Human intersectin 1 (ITSN1) and its paralogue intersectin 2 (ITSN2) are widely expressed proteins which contain two Eps 15 homology (EH) domains, a coiled-coil domain, and five SH3 domains. Intersectins occur in two main isoforms produced by alternative splicing resulting in a short and a long isoform sharing the same protein domain structure. The $\mathrm{COOH}$-terminal segment of the long isoform, which is lacking the short isoform, is composed of a Dbl-homology (DH)

Correspondence to: Dr Markus Seifert, Department of Dermatology, The Saarland University Hospital, 66421 Homburg/Saar, Germany E-mail: hgmsei@uniklinik-saarland.de

Key words: alternative splicing, endocytosis, adaptor proteins, brain gene expression domain, a pleckstrin homology (PH) domain, and a C2domain (1). Intersectin orthologs have been identified in Xenopus (2), Drosophila (3), mouse (4) and rat (5) indicating a highly conserved function. Their biochemical and functional characterization has revealed these multimodular proteins to play an integral role in clathrin-mediated endocytosis (2-5). Clathrin-mediated endocytosis is a multistep process which requires the interaction of more than 20 proteins to form a macromolecular complex. It has major importance in the internalization of receptor-ligand complexes, synaptic vesicle recycling in neurons, and the maintenance of membrane identity in all cells (reviewed in ref. 6,7). The effects of intersectins on endocytosis are derived from its interactions with numerous components of the endocytic pathway including, e.g. clathrin (8), AP2, the AP2-binding protein Epsin 15 (4,5) (Eps15, another EH domain containing endocytic protein), and the endocytic modulators Synaptojanin and Dynamin (5). Thus intersectin appears to act as an adaptor or scaffold for the assembly of the multiprotein complex required for clathrin-mediated endocytosis. As a specific function, ITSN2 was identified to cooperate with WASp and Cdc42 to link the clathrin endocytic machinery to occupancy-induced T-cell receptor endocytosis (9). Like ITSN1, the long isoform of Intersectin 2 (ITSN2-L) exhibits GEF activity, activates Cdc42 and associates with WASp (10) in T-cells. T-cell receptor (TCR) engagement stimulates Cdc42 activation and leads to WASp translocation to endocytic vesicles, where WASp then colocalizes with intersectin 2-L (9).

Human ITSN1 is expressed as three main transcripts of approximately 15,6 and $4.5 \mathrm{~kb}$, with the longest one corresponding to the long isoform expressed almost exclusively in brain, whereas the 6- and 4.5-kb transcripts represent the short isoform $(11,12)$. Human ITSN2 consists of 41 exons that span at least $157 \mathrm{~kb}$ of the genomic DNA. ITSN2 is also expressed as three major transcripts of approximately 10, 8 and $6.5 \mathrm{~kb}$, the long isoform corresponding to the 6.5- and $10-\mathrm{kb}$ transcripts (1). In contrast to ITSN1, ITSN2 major transcripts have a more ubiquitous tissue expression pattern. Isolation and sequencing of human ITSN2 cDNA demonstrated the three major isoforms being produced by alternative splicing (Fig. 1) and revealed some minor splice variants affecting different exons (13). 


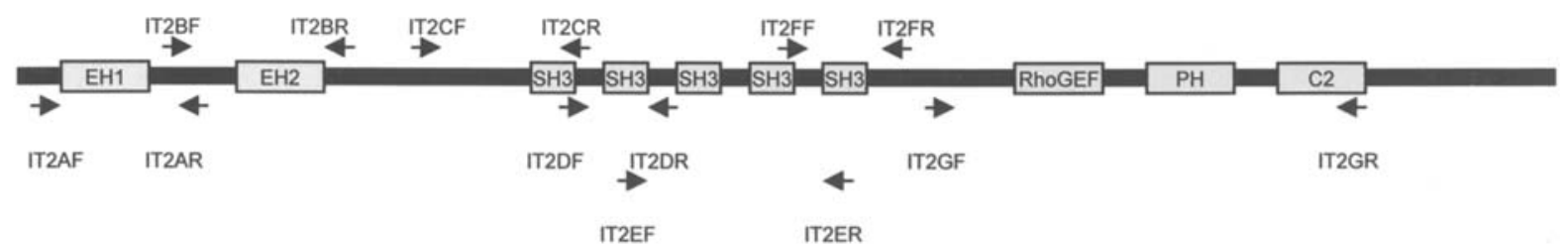

Figure 1. Schematic diagram of the ITSN2 protein indicating different protein domains and localization of the respective primers used for expression analysis. The primer pairs were designed to selectively amplify the different protein domain regions each.

To further analyze ITSN2 expression, especially of the minor variants, we performed RT-PCR analyses using protein domain specific primers for ITSN2 in an adult tissue panel. We report the isolation of new splice variants as well as differential expression of ITSN2 splice variants in human brain.

\section{Materials and methods}

Normal human tissues for tissue panel analyses as well as tumor tissues were collected from patients of the Department of Surgery and the Department of Neurosurgery of Saarland University, Homburg/Saar, Germany, who underwent surgery for a malignant tumor between 1993 and 1996. Informed consent for research use of tissue material was obtained.

The tissue specimens were immediately fresh frozen in liquid nitrogen and stored at $-80^{\circ} \mathrm{C}$ until RNA extraction.

Normal tissue panel. Normal human tissues were derived from single specimens of lung, oesophagus, stomach, colon, liver, spleen, kidney, thymus, and placenta of different individual patients each. Three specimens of normal human brain tissue were analyzed.

Tumor tissues. Malignant tumor tissue was analyzed from lung cancer and oligodendroglioma. The analysis was performed in four specimens of lung cancer (compared with respective normal lung tissue of the same patient) and three specimens of primary oligodendroglioma.

Cell lines. Further analyses in malignant brain tumors included three primary cell lines of oligodendrogliomas and three cell lines of oligodendrogliomas after SCID mouse passage (TX).

Cell culture. Primary cell cultures were established in three oligodendroglioma specimens. After mechanical dissection, primary tissue material was seeded in culture flasks and cells allowed to outgrow from tissue particles to confluency in Dulbecco's modified Eagle's medium (DMEM, GibcoBRL Div. of Invitrogen, Gaithersburg, USA) supplemented with 10\% FCS (PAA, Pasching, Austria), 1x penicillin/streptomycin (GibcoBRL Div. of Invitrogen), and 1x non-essential amino acids (Gibco BRL Div. of Invitrogen) under standard cellculture conditions. TX-cell lines were subcultivated using the identical culture conditions. After one to three culture passages (primary cell lines) or at high passages (TX-cell lines after SCID mouse passage) mRNA extraction was performed in confluent cell cultures followed by the removal of culture media.
RNA extraction. Total RNA was isolated from frozen tissues by the guanidium thiocyanate extraction method as described by Chomczynski et al (14). Afterwards the total RNA was purified using the RNeasy minikit with DNAse treatment according to the manufacturer's instructions (Qiagen, Hilden, Germany) to exclude DNA contamination. In primary cell cultures total RNA was extracted starting with the RNeasy minikit procedure like in frozen tissues. The integrity of all tested total RNA samples was checked by agarose gelelectrophoresis.

Reverse transcription and PCR conditions. Samples containing $2 \mu \mathrm{g}$ of total RNA in a final volume of $20 \mu \mathrm{l}$ were reversetranscribed by AMV reverse transcriptase (Promega, Madison, WI, USA) and an oligo $\mathrm{d}(\mathrm{T})_{15}$ primer, according to the recommendations of the manufacturer.

Amplification of ITSN2 splice variants was performed using $2 \mu \mathrm{l}$ of cDNA reaction in a final volume of $50 \mu \mathrm{l}$ containing $1 \mathrm{X}$ reaction buffer for Taq Polymerase (Amersham Pharmacia, Freiburg, Germany), 15 pmol of each primer [IT2A-F 5'-GAGTCGCGCTGTGACGAG-3', IT2A-R 5'-GA TGACCCATGAGGCAATGT-3'; IT2B-F 5'-TTCAGCCTTT ACCCATTCCTT-3', IT2B-R 5'-GTTCCCTCGCTCATAGT TGG-3'; IT2C-F 5'-GAAGCACTGAATGGCAAACA-3', IT2C-R 5'-TTTCCTTGCAGCCTCATCTT-3'; IT2D-F 5'AGGAAAGCAAAGCAAGGAAA-3', IT2D-R 5'-TAAAGC CAACCAGGTTCTCC-3'; IT2E-F 5'-TACCCCTTTGAAGC AAGGAA-3', IT2E-R 5'-TCCTGGTGCAAGGCTAAGTT-3'; IT2F-F 5'-GGAGAGTGGTGGACAGGAAG-3', IT2F-R 5'ACAGGATGAAAGGCAGGTGT-3'; IT2G-F 5'-GATGATC CTGATTGGTGGC-3', IT2G-R 5'-CAAAAAGCTGCAGGT CAAA-3'; B2MFor 5'-CCAGCAGAGAATGGAAAGTC-3', ß2MRev 5'-GATGCTGCTTACATGTCTC-3' (15)]; $100 \mathrm{mM}$ of each deoxynucleotide triphosphate and $2 \mathrm{U}$ Taq DNA Polymerase (Amersham Pharmacia). PCR was carried out for 35 amplification cycles consisting of denaturation at $95^{\circ} \mathrm{C}$ for $15 \mathrm{sec}$, primer annealing at $60^{\circ} \mathrm{C}$ for $30 \mathrm{sec}$, extension at $72^{\circ} \mathrm{C}$ for $45 \mathrm{sec}$ and a final extension time of $7 \mathrm{~min}$ at $72^{\circ} \mathrm{C}$. The amplified products were visualized by agarose-gelelectrophoresis and subsequently cloned in the pCR4-TOPO (Invitrogen, San Diego, CA, USA) vector, sequenced and the splice junctions were identified by comparing the sequences of the amplified products with ITSN2 cDNA (NM_006277, NM_019595, NM_147152).

Nucleotide sequence analysis. Nucleotide sequences of splice variants were determined by the dideoxy chain-termination method using a BigDye Terminator Cycle sequencing kit (Applied Biosystems, Foster City, CA, USA). The sequencing 


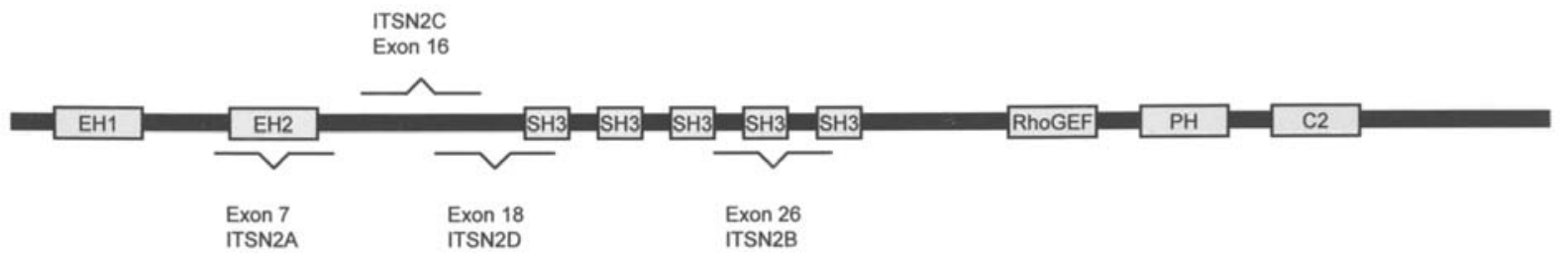

Figure 2. Schematic diagram of the ITSN2 protein showing the different protein domains with regard to the differentially spliced exons of the ITSN2 splice variants.
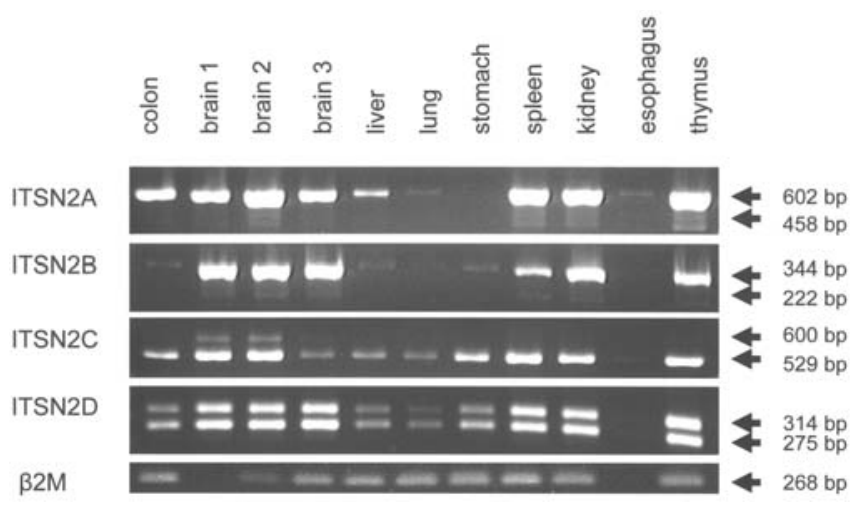

Figure 3. Gel electrophoresis of ITSN2 splice variants obtained by RT-PCR in different human tissues (colon, brain liver, lung, stomach, spleen, kidney, oesophagus, and thymus). The fragment size of the RT-PCR products is indicated at the right margin. The respective RT-PCR results of the B2Microglobulin housekeeping gene is demonstrated.

run was performed in a genetic analyzer (model 310, Applied Biosystems). The sequence homology of the retrieved DNA sequences was examined employing the Sequencher ${ }^{\mathrm{TM}}$ DNA sequencing program (Gene Codes Corporation, Ann Arbor, MI, USA).

\section{Results}

Analysis of ITSN2 splice variants. To determine the sequence of novel and previously described splice variants, total cellular RNA isolated from various tissues was subjected to RT-PCR. Yielding a more complex result a set of primers spanning different regions (Fig. 1) of ITSN2 was used. Sequence analysis of the subcloned cDNAs and comparison with the known ITSN2 sequence demonstrated the isolation of four ITSN2 minor splice variants (Fig. 2). Thereby two of them could be identified as new, formerly undescribed ITSN2 minor splice variants (termed ITSN2A and B). Furthermore two already known splice variants (termed ITSN2C and D) previously described by Pucharcos et al (13) were detected. All four variants have intact reading frames and no alternative translation stop indicating their functional relevance. The new variant ITSN2A lacks exon 7. The sequence analysis of the resulting protein using the simple modular architecture research tool (SMART, http://smart.embl-heidelberg.de/) shows that this affects part of the EH2 domain (Fig. 2), however it will not totally eliminate it. ITSN2B, the second new variant lacks exon 26 and differs from the previously described minor variant, in which exon 26 and exon 27 are missed in combination (13). Sequence analysis (SMART)

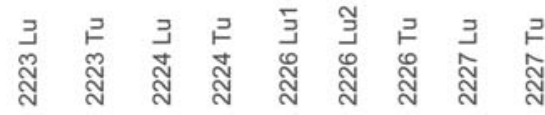

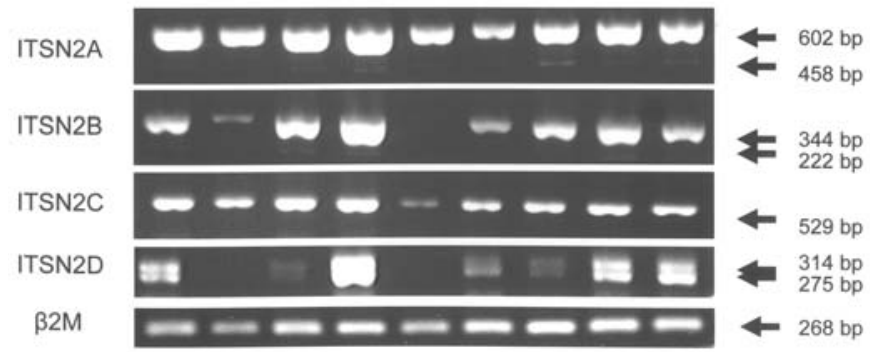

Figure 4. Gel electrophoresis of ITSN2 splice variants obtained by RT-PCR in tissues from lung tumors (Tu) and corresponding normal lung ( $\mathrm{Lu}$ ). Normal lung specimens were obtained from normal lung of the respective lung tumor patients. In case 2226 two different normal lung specimens were analyzed.

showed that the alternative splicing of exon 26 is sufficient to eliminate one of the five SH3 domains (SH3D).

By the adult normal tissue panel analysis ITSN2A and B variants were observed in all analyzed tissues (Fig. 3). The fragment intensity, though, was seemingly weaker in specimens from liver, lung, and stomach; real-time PCR, however, was not performed to reliably quantify possible tissue-specific expression differences. Comparative expression analysis in normal human lung and in lung tumors did not demonstrate differential expression for ITSN2A and B minor variants (Fig. 4).

In the ITSN2D minor variant previously described by Pucharcos et al (13) a short fragment of 13 amino acids inside exon 18 is spliced. In tissue panel analysis ITSN2D expression was demonstrated in all analyzed normal tissues without a tissue-specific expression pattern (Fig. 3). Concerning the two new minor variants also in ITSN2D no evident expression difference was observed between normal human lung and lung tumor tissues (Fig. 4).

In contrast, regarding the ITSN2C minor variant a tissuespecific expression of ITSN2C was demonstrated, being restricted to normal human brain. The ITSN2C variant includes exon 16 and by this differs from the mainly transcribed variants lacking this specific exon.

To selectively analyze the expression of exon 16 we performed RT-PCR using exon 16 flanking primers, yielding a full-length transcript of $600 \mathrm{bp}$ (including exon 16) or a spliced transcript of 510 bp lacking the $90 \mathrm{bp}$ of exon 16 . Thereby, remarkably, the exon 16 containing variant was almost exclusively shown in normal human brain (Fig. 3). 

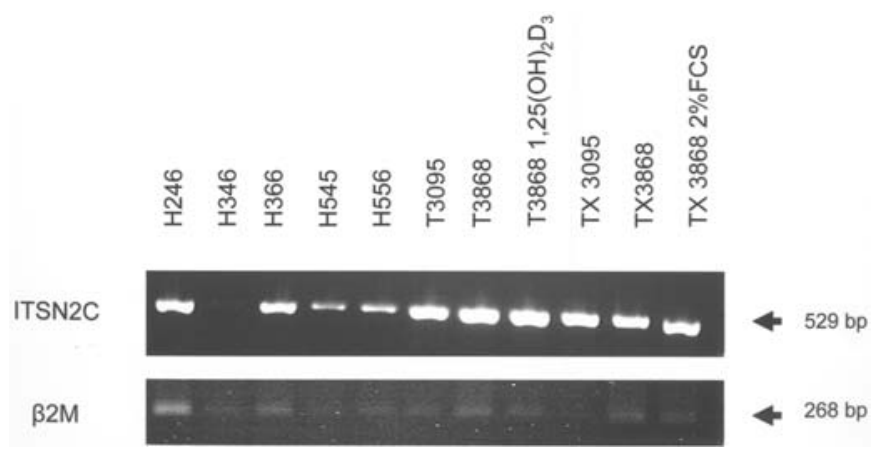

Figure 5. Gel electrophoresis of ITSN2 splice variants obtained by RT-PCR in tissues from human oligodendroglioma (H246, H346, H366, H545, H556), oligodendroglioma primary cell lines (T3095, T3868), and oligodendroglioma cell lines after SCID mouse passage (TX-cell lines). From one primary cell line (T3868) and one TX-cell line additional analyses after cell culture modification (vitamin D-supply or change of the FCS-concentration) revealed similar results each.

Using exon 16 specific primers a weak expression of the exon 16 complete transcript (ITSN2C; full-length transcript) was also detected in all other analyzed tissues; though, it was still clearly underrepresented compared to the exon 16-lacking shorter transcript. Thus, with the exception of brain tissue, we demonstrated that ITSN2C is only very weakly expressed in the majority of analyzed tissues.

Sequence analysis of exon 16 showed a high rate of hydrophobic amino acids hinting to a possible function as transmembrane domain. While using the SMART program no homology to such a domain was identified, other programs such as ConPred II (http://bioinfo.si.hirosaki-u.ac.jp/ $\sim$ ConPred2/) indicated a transmembrane (TM) domain comprising AA 627-647 (NP_006268). In contrast, exon 16-lacking isoforms (NP_062541) did not reveal any TM domains. AA 621-647 of the ITSN2 are encoded by exon 16 demonstrating exon 16 as the only exclusive TM-domain of the ITSN2-protein.

On the basis of ITSN2C expression in normal human brain the expression analysis was extended to human brain tumor tissues as well as human brain tumor cell lines (oligodendrogliomas).

Using the above described exon 16 flanking primers unlike normal human brain tissue ITSN2 $\mathrm{C}$ was not detected in brain tumor tissues or in tumor cell lines, whereas the exon 16-lacking major variants were detected in all samples (Fig. 5). Comparing the expression of ITSN2C between normal human lung and four lung tumor tissues did not reveal differential expression, showing similar expression in lung tumors as in the corresponding normal tissue (Fig. 4).

\section{Discussion}

Two new ITSN2 minor splice variants were described, which are able to produce a respective protein product. This adds to the rare information on ITSN2 expression. Four minor ITSN2 splice variants, the two newly detected and two formerly reported variants (13), were analyzed regarding the potential tissue specificity of their expression. Three of them (ITSN2A, $\mathrm{B}$, and D) revealed no tissue-specific expression pattern and no expression differences were observed between normal lung and lung tumor tissues. At least regarding lung tumor, these described minor variants therefore do not seem to be involved in tumor-associated changes.

In contrast, regarding the ITSN2C minor variant several remarkable results were obtained, deviating from the above described other minor splice variants.

Interestingly, the complete ITSN2 transcript containing all exons is not the major isoform that is expressed. In the overall expression of ITSN2 exon sparing splice variants represent the minor expression variants. This is different regarding exon 16 , which is almost exclusively spliced in the ITSN2 major transcripts. ITSN2C is defined as a minor splice variant including exon 16. Unlike the above described minor variants, for the most important, tissue-specific expression of ITSN2C in normal human brain was demonstrated.

The relatively strong selective expression in normal human brain raised the suggestion, that the exon 16-comprising minor variant (ITSN2C) may have a brain-specific function.

In RNA from human brain tumors different from total human brain RNA ITSN2C was not demonstrated. Thereby the relative lack of ITSN2C was also observed in brain tumor cell lines (primary cell lines, cell lines after SCID mouse passage) as in uncultivated brain tumor tissues, thus excluding a cell culture induced change of expression. Oligodendrogliomas are human brain derived tumors. The malignant cell type is of glia cell origion. Glia cells are non-neuronal networking cells interspersed to the neurons. Thus, the proof of ITSN2C in total brain but not in oligodendroglioma may be due to primarily neuronal expression. The tissue-specific selective relative overrepresentation of this minor splice variant may hint to a neuron-specific role of ITSN2C. On the other hand loss of ITSN2C expression in glial tumors versus normal glia cells was also possible; thereby a physiologic role of ITSN2C in glia cells might be suggested. Further investigation is needed to clearly identify the cellular source and function of ITSN2C in human brain tissue.

\section{Acknowledgements}

This study was supported by the Stefan Morsch Stiftung.

\section{References}

1. Pucharcos $C$, Estivill $X$ and de la Luna $S$ : Intersectin 2, a new multimodular protein involved in clathrin-mediated Endocytosis. FEBS Lett 478: 43-51, 2000.

2. Yamabhai M, Hoffman NG, Hardison NL, McPherson PS, Castagnoli L, Cesareni G and Kay BK: Intersectin, a novel adaptor protein with two Eps15 homology and five Src homology 3 domains. J Biol Chem 273: 31401-31407, 1998.

3. Roos J and Kelly RB: Dap160, a neural-specific Eps15 homology and multiple $\mathrm{SH} 3$ domain-containing protein that interacts with Drosophila dynamin. J Biol Chem 273: 19108-19119, 1998.

4. Sengar AS, Wang W, Bishay J, Cohen S and Egan SE: The EH and $\mathrm{SH} 3$ domain Ese proteins regulate endocytosis by linking to dynamin and Eps15. EMBO J 18: 1159-1171, 1999.

5. Okamoto M, Schoch S and Sudhof TC: EHSH1/intersectin, a protein that contains $\mathrm{EH}$ and $\mathrm{SH} 3$ domains and binds to dynamin and SNAP-25. A protein connection between exocytosis and endocytosis? J Biol Chem 274: 18446-18454, 1999.

6. Conner SD and Schmid SL: Regulated portals of entry into the cell. Nature 422: 37-44, 2003.

7. Traub LM: Sorting it out: AP-2 and alternate clathrin adaptors in endocytic cargo selection. J Cell Biol 163: 203-208, 2003. 
8. Hussain NK, Yamabhai M, Ramjaun AR, Guy AM, Baranes D, O'Bryan JP, Der CJ, Kay BK and McPherson PS: Splice variants of intersectin are components of the endocytic machinery in neurons and nonneuronal cells. J Biol Chem 274: 15671-15677, 1999.

9. McGavin MK, Badour K, Hardy LA, Kubiseski TJ, Zhang J and Siminovitch KA: The intersectin 2 adaptor links Wiskott Aldrich Syndrome protein (WASp)-mediated actin polymerization to T cell antigen receptor endocytosis. J Exp Med 194: 1777-1787, 2001.

10. Hussain NK, Jenna S, Glogauer M, Quinn CC, Wasiak S, Guipponi M, Antonarakis SE, Kay BK, Stossel TP, Lamarche-Vane $\mathrm{N}$ and McPherson PS: Endocytic protein intersectin-l regulates actin assembly via Cdc42 and N-WASP. Nat Cell Biol 3: 927-932, 2001.

11. Guipponi M, Scott HS, Chen H, Schebesta A, Rossier C and Antonarakis SE: Two isoforms of a human intersectin (ITSN) protein are produced by brain-specific alternative splicing in a stop codon. Genomics 53: 369-376, 1998.
12. Guipponi M, Scott HS, Hattori M, Ishii K, Sakaki Y and Antonarakis SE: Genomic structure, sequence, and refined mapping of the human intersectin gene (ITSN), which encompasses $250 \mathrm{~kb}$ on chromosome $21 \mathrm{q} 22.1->\mathrm{q} 22.2$. Cytogenet Cell Genet 83: 218-220, 1998.

13. Pucharcos C, Casas C, Nadal M, Estivill X and de la Luna S: The human intersectin genes and their spliced variants are differentially expressed. Biochim Biophys Acta 1521: 1-11, 2001.

14. Chomczynski P and Sacchi N: Single-step method of RNA isolation by acid guanidinium thiocyanate-phenol-chloroform extraction. Anal Biochem 162: 156-159, 1987.

15. Schneider-Stock R, Boltze C, Jager V, Eppelen J, Landt O, Peters B, Rys J and Roessner A: Elevated telomerase activity, cMyc, and hTERT mRNA expression: associated with tumor progression in malignant lipomatous tumours. J Pathol 199: 517-525, 2003. 